\title{
Función sigmoide como modelo de predicción para riego en trigo (Triticum aestivum), Distrito 014, Mexicali, Baja California
}

\author{
Sigmoid function as prediction model for irrigation in wheat \\ (Triticum aestivum), District 014, Mexicali, Baja California
}

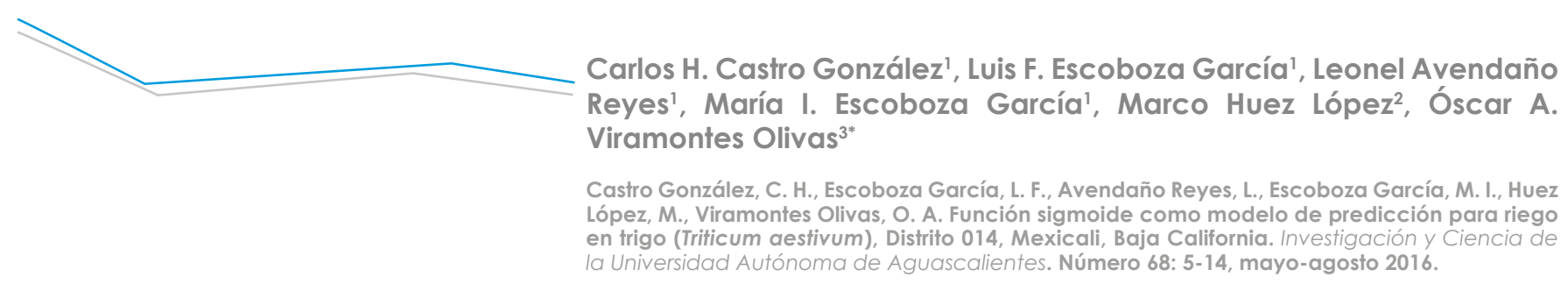

\section{RESUMEN}

Para disponer del recurso hídrico en el Distrito de Riego 014, Río Colorado y llevar a cabo los riegos en los cultivos, se cumple con una programación basada en planes de riego que verifican avances, cambios y ajustes periódicos. Con el fin de reforzar la programación, se utilizaron métodos matemáticos para predecir la sistematización de riegos en trigo (Triticum aestivum). Se emplearon tres modelos de función sigmoide, para los cuales se usaron datos experimentales observados y acumulados en la superficie agrícola del DR 014 en Mexicali y San Luis Río Colorado, Sonora, durante el ciclo 2010-2011. La validación de los modelos fue mediante el logístico de Verhulst, función Gompertz y Beta de crecimiento. Los parámetros como pruebas de bondad de ajuste, verificación de linealidad, análisis de varianza y residual se estimaron con el programa especializado para regresiones no lineales. De acuerdo con los resultados, los modelos Verhulst y Gompertz describieron el comportamiento de la superficie regada en tiempo,

Palabras clave: irrigación, parcelas, producción, programación.

Keywords: irrigation, plots, production, programming.

Recibido: 15 de abril de 2015, aceptado: 28 de enero de 2016

Instituto de Ciencias Agrícolas, Universidad Autónoma de Baja California.

Universidad de Sonora.

3 Facultad de Contaduría y Administración, Universidad Autónoma de Chihuahua campus Universitario 2.

* Autor para correspondencia: violioscar@gmail.com, oviramon@uach.mx por lo que estadísticamente se considera confiable para fines de predicción en la elaboración de planes de riego.

ABSTRACT

To have the water resources in the Irrigation District 014, Colorado River and carry out irrigation on crops is met based programming irrigation schemes that verify progress, changes and periodic adjustments. In order to strengthen the programming, mathematical methods were used to predict the systematization of irrigation in wheat (Triticum aestivum). Three models of sigmoid function for which observed experimental data were used and accumulated in the agricultural area of the DR 014 in Mexicali and San Luis Rio Colorado, Sonora, during the 2010-2011 cycle were used. The model validation was using the Verhulst logistic, Gompertz function and growth Beta. Parameters such as goodness of fit tests, linearity verification, analysis of variance and residual, were estimated with the specialized program for nonlinear regressions. According to the results, Gompertz and Verhulst models described the behavior of the irrigated surface in time, so statistically it is considered reliable for forecasting purposes in developing irrigation schemes.

INTRODUCCIÓN

Cortez Lara (2011) menciona que en los valles de Mexicali, Baja California y San Luis Río Colorado, Sonora, se cultiva bajo riego una superficie de 123,000 


\section{InVEStIGRCIÓn

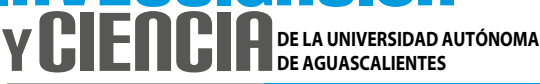

haconaguaporgravedad; 47 y 20,000 ha consistemas de pozos federal y particular, respectivamente. La cédula de cultivos comúnmente establecida es de 96,000 ha (trigo); 33,000 ha (algodón) y 32,000 ha de alfalfa y otros cultivos como hortalizas, frutales y sorgo. Para este tipo de riego, el manejo se realiza con obras hidráulicas e infraestructura del Distrito de Riego 014, Río Colorado y con el suministro de agua del acuífero a través de una batería de 640 pozos profundos (CONAGUA, 2010), en donde al trigo se le aplican cinco riegos con una lámina neta de 90 $\mathrm{cm}$ del mes de octubre a enero (2010-2011) y dentro de una temporada de mediados de octubre a la tercera semana de abril de 2011. La cosecha es a partir del 10 de mayo (PRSG, 2011).

Para el establecimiento de cultivos, el trabajo se basa en un plan de riegos de acuerdo a la CONAGUA (2009), el cual debe fundamentarse técnicamente en las necesidades hídricas de los mismos y cumplir con las restricciones propias del Tratado de Aguas de 1944 (SRE, 1944) que rige el manejo del agua del río Colorado. Este proceso se debe desarrollar de manera racional, ya que la presión y demanda del recurso es cada vez mayor por parte de los usuarios urbanos, industriales y agrícolas con una disponibilidad fija con tendencias a la baja por efectos climáticos y la sequía presente en su cuenca, por lo que será necesario mejorar los métodos y metodologías para el uso más eficiente del recurso (Sánchez, 2004).

Cortez Lara (2005) comenta que cuando los cultivos ya están establecidos en los valles de Mexicali y San Luis Río Colorado, los organismos encargados de la distribución y entrega del agua a los usuarios elaboran gráficas que representan la superficie regada acumulada en el tiempo para observar avances y comparar con lo programado con el fin de realizar cambios y ajustes en las demandas hídricas. Este comportamiento gráfico se representa con una curva sigmoidea según lo expone Kirchhoff (2012) y puede ser simulada con modelos empíricos de la función sigmoide o logística (Yin et al., 2003) que al alcanzar sus ecuaciones y parámetros pueden ser utilizados con fines de predicción en la elaboración de planes de riego expresando la superficie regada acumulada, como fracción del área total establecida de un cultivo, se obtienen los índices de riego que representan de manera unitaria, extrapolar cualquier dimensión con fines de programación (Alonso Báez et al., 2003).
El objetivo de esta investigación fue evaluar tres modelos de predicción: función Gompertz, modelo Logístico de Verhulst y función Beta de Crecimiento (Yin et al., 2003) para determinar y planear de manera racional el riego del cultivo de trigo Triticum aestivum, para ajustarlo a los datos observados en la superficie regada durante el ciclo 2010-2011 en el Distrito de Riego 014, Mexicali, Baja California.

\section{MATERIALES Y MÉTODOS}

Se recurrió a la información hidroagrícola del Distrito de Riego 014, Río Colorado S.R.L. de I.P. de C.V., referente al ciclo agrícola 2010-2011 del cultivo de trigo establecido dentro del sistema de agua por gravedad, con una superficie de 63,161 ha físicas y 325,725 ha de riego, cuya información se concentra en la Tabla 1.

Las hectáreas de riego se refieren a la suma de las hectáreas físicas que se riegan en cada ciclo. Para el análisis del estudio los datos de las regadas acumuladas fueron expresados como fracción de superficie total establecida y se definió a dicha fracción como Índice de Riego (IR) que, para este estudio, es el valor entre 0 y 1 que representa una cantidad de hectáreas regadas en determinado tiempo, siendo $I R=1$ cuando se riega la superficie total establecida. La unidad de tiempo utilizada fue la decena (10 d), los índices decenales y por riego se muestran en la Tabla 2. Para efectos de cálculo de parámetros y análisis estadísticos, los riegos se inician con la Decena 1, ya que estos fueron analizados de forma independiente.

Al graficar la información de la superficie regada acumulada de la Tabla 1 se obtiene una serie de curvas de tipo sigmoideo, como se aprecia en la Figura 1.

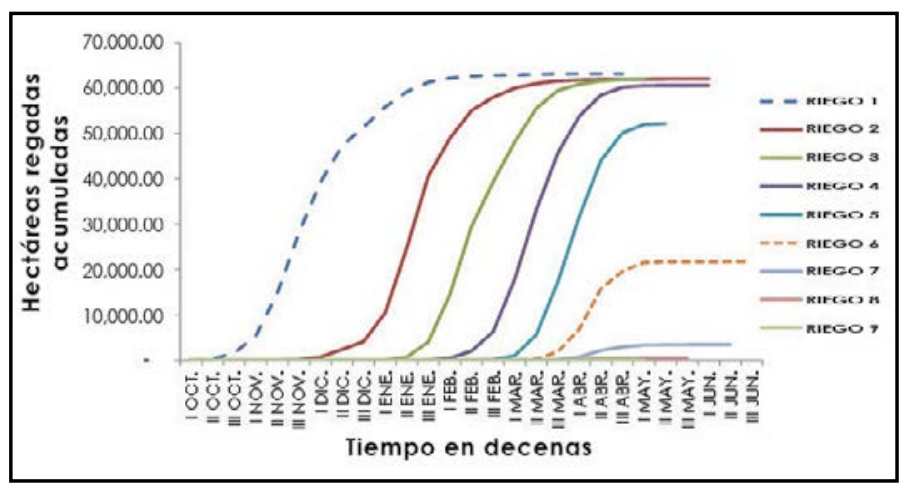

Figura 1. Superficie regada acumulada por riego de trigo 2010-2011. Elaboración propia. 


\section{InVESEIGACIÓn

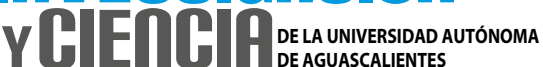 \\ DE AGUASCALIENTES}

Tabla 1. Superficie acumulada regada por decena en el ciclo de trigo 2010-2011

\begin{tabular}{|c|c|c|c|c|c|c|c|c|c|}
\hline $\begin{array}{c}\text { Decena del } \\
\text { mes }\end{array}$ & $\begin{array}{c}\text { Riego } \\
1\end{array}$ & $\begin{array}{c}\text { Riego } \\
2\end{array}$ & $\begin{array}{c}\text { Riego } \\
3\end{array}$ & $\begin{array}{c}\text { Riego } \\
4\end{array}$ & $\begin{array}{c}\text { Riego } \\
5\end{array}$ & $\begin{array}{c}\text { Riego } \\
6\end{array}$ & $\begin{array}{c}\text { Riego } \\
7\end{array}$ & $\begin{array}{c}\text { Riego } \\
8 \\
\end{array}$ & $\begin{array}{c}\text { Riego } \\
9\end{array}$ \\
\hline I Oct. & 34.00 & - & - & - & - & - & - & - & - \\
\hline II Oct. & 236.73 & - & - & - & - & - & - & - & - \\
\hline III Oct. & $1,578.73$ & 13.00 & - & - & - & - & - & - & - \\
\hline I Nov. & $5,396.51$ & 26.00 & - & - & - & - & - & - & - \\
\hline II Nov. & $14,803.35$ & 79.00 & - & - & - & - & - & - & - \\
\hline III Nov. & $28,197.57$ & 152.00 & - & - & - & - & - & - & - \\
\hline I Dic. & $39,324.63$ & 686.66 & 6.00 & - & - & - & - & - & - \\
\hline II Dic. & $47,282.29$ & $2,316.81$ & 6.00 & - & - & - & - & - & - \\
\hline III Dic. & $51,507.89$ & $4,140.11$ & 16.00 & 24.00 & - & - & - & - & - \\
\hline I Ene. & $55,858.12$ & $10,475.64$ & 66.00 & 24.00 & - & - & - & - & - \\
\hline II Ene. & $59,268.30$ & $24,790.91$ & 651.50 & 24.00 & - & - & - & - & - \\
\hline III Ene. & $61,354.27$ & $40,694.02$ & $4,074.03$ & 66.00 & - & - & - & - & - \\
\hline I Feb. & $62,248.08$ & $48,996.56$ & $14,522.75$ & 443.35 & - & - & - & - & - \\
\hline II Feb. & $62,693.87$ & $55,067.50$ & $29,445.81$ & $1,998.05$ & 30.00 & - & - & - & - \\
\hline III Feb. & $62,883.87$ & $58,005.95$ & $39,385.30$ & $6,276.60$ & 144.85 & - & - & - & - \\
\hline I Mar. & $62,965.87$ & $60,059.64$ & $48,139.03$ & $17,853.68$ & 995.60 & 14.00 & - & - & - \\
\hline II Mar. & $63,059.07$ & $61,040.00$ & $55,548.17$ & $33,108.22$ & $5,563.66$ & 284.65 & 12.00 & 64.00 & - \\
\hline III Mar. & $63,136.57$ & $61,649.96$ & $59,528.00$ & $45,763.40$ & $17,293.81$ & $1,850.45$ & 137.00 & 64.00 & - \\
\hline I Abr. & $63,152.07$ & $61,862.06$ & $60,943.40$ & $53,867.38$ & $31,792.52$ & $6,916.78$ & 705.86 & 170.00 & - \\
\hline II Abr. & $63,152.07$ & $61,938.06$ & $61,646.65$ & $58,522.59$ & $44,066.73$ & $15,740.23$ & $2,141.21$ & 353.00 & 5.00 \\
\hline III Abr. & $63,160.57$ & $61,975.56$ & $61,933.05$ & $60,252.65$ & $50,193.77$ & $19,676.81$ & $2,937.60$ & 413.00 & 25.00 \\
\hline I May. & & $61,990.56$ & $62,008.05$ & $60,587.65$ & $52,029.80$ & $21,555.93$ & $3,349.60$ & 472.00 & 27.00 \\
\hline II May. & & $62,000.06$ & & $60,626.65$ & $52,252.80$ & $21,689.43$ & $3,407.40$ & 488.00 & \\
\hline III May. & & $62,008.06$ & & $60,629.65$ & & $21,689.43$ & $3,415.40$ & 498.00 & \\
\hline I Jun. & & $62,010.06$ & & $60,636.65$ & & $21,700.43$ & $3,415.40$ & & \\
\hline II Jun. & & & & & & $21,708.43$ & $3,421.40$ & & \\
\hline III Jun. & & & & & & $21,710.43$ & & & \\
\hline Ha por riego & 63,161 & 62,010 & 62,008 & 60,637 & 52,253 & 21,710 & 3,421 & 498 & 27 \\
\hline
\end{tabular}

Tabla 2. Índices de riego (IR), decenales y globales del cultivo de trigo 2010-201 1

\begin{tabular}{|c|c|c|c|c|c|c|c|c|c|c|c|}
\hline Decenas & $\begin{array}{c}\text { Riego } \\
1\end{array}$ & $\begin{array}{c}\text { Riego } \\
2\end{array}$ & $\begin{array}{c}\text { Riego } \\
3\end{array}$ & $\begin{array}{c}\text { Riego } \\
4\end{array}$ & $\begin{array}{c}\text { Riego } \\
5\end{array}$ & $\begin{array}{c}\text { Riego } \\
6\end{array}$ & $\begin{array}{c}\text { Riego } \\
7\end{array}$ & $\begin{array}{c}\text { Riego } \\
8\end{array}$ & $\begin{array}{c}\text { Riego } \\
9\end{array}$ & IR global & Ha riego \\
\hline 1 & 0.00054 & 0.00021 & 0.00009 & 0.000380 & 0.00047 & 0.00022 & 0.00019 & 0.00101 & 0.00008 & & \\
\hline 2 & 0.00375 & 0.00041 & 0.00009 & 0.000380 & 0.00229 & 0.00451 & 0.00217 & 0.00101 & 0.00040 & & \\
\hline 3 & 0.02500 & 0.00125 & 0.00025 & 0.000380 & 0.01576 & 0.02930 & 0.01118 & 0.00269 & 0.00043 & & \\
\hline 4 & 0.08544 & 0.00241 & 0.00104 & 0.001045 & 0.08809 & 0.10951 & 0.03390 & 0.00559 & & & \\
\hline 5 & 0.23438 & 0.01087 & 0.01031 & 0.007019 & 0.27381 & 0.24921 & 0.04651 & 0.00654 & & & \\
\hline 6 & 0.44644 & 0.03668 & 0.06450 & 0.031634 & 0.50336 & 0.31154 & 0.05303 & 0.00747 & & & \\
\hline 7 & 0.62261 & 0.06555 & 0.22993 & 0.099375 & 0.69769 & 0.34129 & 0.05395 & 0.00773 & & & \\
\hline 8 & 0.74860 & 0.16586 & 0.46621 & 0.282671 & 0.79470 & 0.34340 & 0.05407 & 0.00788 & & & \\
\hline 9 & 0.81551 & 0.39251 & 0.62357 & 0.524191 & 0.82377 & 0.34340 & 0.05407 & & & & \\
\hline 10 & 0.88438 & 0.64429 & 0.76217 & 0.724556 & 0.82730 & 0.34358 & 0.05417 & & & & \\
\hline 11 & 0.93838 & 0.77575 & 0.87948 & 0.852864 & & 0.34370 & & & & & \\
\hline 12 & 0.97140 & 0.87187 & 0.94249 & 0.926568 & & 0.34373 & & & & & \\
\hline 13 & 0.98555 & 0.91839 & 0.96490 & 0.953960 & & & & & & & \\
\hline 14 & 0.99261 & 0.95090 & 0.97603 & 0.959264 & & & & & & & \\
\hline 15 & 0.99562 & 0.96643 & 0.98057 & 0.959881 & & & & & & & \\
\hline 16 & 0.99692 & 0.97608 & 0.98175 & 0.959929 & & & & & & & \\
\hline 17 & 0.99839 & 0.97944 & & 0.960040 & & & & & & & \\
\hline 18 & 0.99962 & 0.98064 & & & & & & & & & \\
\hline 19 & 0.99987 & 0.98124 & & & & & & & & & \\
\hline 20 & 0.99987 & 0.98148 & & & & & & & & & \\
\hline 21 & 1.00000 & 0.98163 & & & & & & & & & \\
\hline 22 & & 0.98175 & & & & & & & & & \\
\hline 23 & & 0.98178 & & & & & & & & & \\
\hline IR, Por riego & 1.00000 & 0.98178 & 0.98175 & 0.96004 & 0.82730 & 0.34373 & 0.05417 & 0.00788 & 0.00043 & 5.15709 & \\
\hline Ha regadas & 63,161 & 62,010 & 62,008 & 60,637 & 52,253 & 21,710 & 3,421 & 498 & 27 & & 325,725 \\
\hline
\end{tabular}

Elaboración propia

El comportamiento de las curvas de riego de la Figura 1 es la parte central del estudio que consistió en obtener el modelo de predicción de la superficie regada acumulada en cada riego, en donde se analizaron los modelos:
1. Modelo logístico de Verhulst: $y=\frac{y \max }{1+e^{-k(t-t i)}}$

2. Función Gompertz: $\quad y=y \max e^{-e^{-k(t-t i)}}$

3. Función beta de crecimiento: $y=y \max \left(1+\frac{t m-t}{t m-t i}\right)\left(\frac{t}{t m}\right)^{\frac{t m}{t m-t i}} \quad$ para $0 \leq t i<t m$ 
Donde $(y)$ Variable dependiente; (ymax) Valor máximo de $y_{;}(k)$ Constante de curvatura del modelo; (t) Tiempo (variable independiente); (ti) Tiempo de inflexión; $(\dagger \mathrm{m})$ Tiempo máximo y $(e)$ Número de Euler.

Para obtener los parámetros en los modelos se recurrió al uso de un programa especializado para regresiones no lineales mediante el procedimiento NLIN de SAS 9.2 con la opción Marquardt-Hougaard. Las variables de entrada fueron los datos observados en cada riego y la expresión matemática de las ecuaciones que además de los parámetros, genera la estimación de límite de confianza al 95\%, error estándar, grado de asimetría (skewness), matriz de correlación y un análisis de varianza para el modelo.

Con los datos observados y estimados en cada uno de los modelos se determinaron los indicadores de bondad de ajuste, tales como: media del error absoluto (MEA), media del cuadrado del error (MCE) y coeficiente de determinación para modelos no lineales $\left(R_{1}{ }^{2}\right)$.

El criterio para la selección del modelo se basó en el cumplimiento de las siguientes restricciones:

- Que el modelo fuera estadísticamente significativo en el análisis de varianza.

- Que los parámetros obtenidos presentaran indicadores aceptables de límites de confianza y asimetría.
- Que el análisis de estimados y observados cumpliera las pruebas de bondad de ajuste de MEA, $M C E$ y $R_{l}^{2}$.

- Que los parámetros de los modelos no fuesen correlacionados.

- Que los modelos cumplieran con los supuestos de normalidad e independencia del residual.

\section{RESULTADOS}

De los nueve riegos aplicados se descartó el último, ya que el porcentaje de aplicación fue muy bajo (0.043\%) con solo tres datos observados, lo que imposibilitó un análisis estadístico confiable en este caso. En la revisión de la información utilizada (Tabla 1) se observa que en la parte final de los riegos este se registra tardíamente, ya que aun cuando la temporada se termina, se siguen ingresando datos. Al margen de estas dos consideraciones se realizó el análisis de la información (Tabla 3), en donde se muestra el concentrado de los resultados del análisis de varianza en los tres modelos propuestos.

En los tres modelos se aprecia que el análisis de varianza de cada uno de ellos y en todos los riegos son altamente significativos, ya que en la fuente de variación atribuida al modelo se obtiene un valor de $(P<.0001)$. Analizando el parámetro $(k)$ de los modelos Gompertz y Verhulst y el parámetro ( $t$ m) del modelo Beta, en la Tabla 4, se confirma con

Tabla 3. Suma de cuadrados y significancia de los modelos propuestos

\begin{tabular}{|c|c|c|c|c|c|c|}
\hline Modelo & S.C. Modelo & S.C. Total & Riego 1 & S.C. Modelo & S.C. Total & Riego 5 \\
\hline & GL= 2 & GL= 21 & Pr $>\mathbf{F}$ & GL= 2 & GL= 10 & Pr $>\mathbf{F}$ \\
\hline Gompertz & 13.41720 & 13.41880 & $<.0001$ & 2.81450 & 2.81770 & $<.0001$ \\
\hline Verhulst & 13.40520 & 13.41880 & $<.0001$ & 2.81700 & 2.81770 & $<.0001$ \\
\hline Beta & 13.21950 & 13.41880 & $<.0001$ & 2.79390 & 2.81770 & $<.0001$ \\
\hline & $\mathbf{G L = 2}$ & $\mathbf{G L = 2 3}$ & Riego 2 & $\mathbf{G L = 2}$ & $\mathbf{G L = 1 2}$ & Riego 6 \\
\hline Gompertz & 12.33410 & 12.33720 & $<.0001$ & 0.87820 & 0.87870 & $<.0001$ \\
\hline Verhulst & 12.33230 & 12.33720 & $<.0001$ & 0.87860 & 0.87870 & $<.0001$ \\
\hline Beta & 11.98630 & 12.33720 & $<.0001$ & 0.85730 & 0.87870 & $<.0001$ \\
\hline & $\mathbf{G L = 2}$ & $\mathbf{G L = 1 6}$ & Riego 3 & $\mathbf{G L = 2}$ & $\mathbf{G L = 1 0}$ & Riego 7 \\
\hline Gompertz & 6.71310 & 6.71500 & $<.0001$ & 0.01790 & 0.01790 & $<.0001$ \\
\hline Verhulst & 6.70620 & 6.71500 & $<.0001$ & 0.01790 & 0.01790 & $<.0001$ \\
\hline Beta & 6.61540 & 6.71500 & $<.0001$ & 0.01760 & 0.01790 & $<.0001$ \\
\hline & $\mathbf{G L = 2}$ & $\mathbf{G L = 1 7}$ & Riego 4 & $\mathbf{G L = 2}$ & $\mathbf{G L = 8}$ & Riego 8 \\
\hline Gompertz & 7.06820 & 7.07120 & $<.0001$ & 0.00026 & 0.00026 & $<.0001$ \\
\hline Verhulst & 7.07000 & 7.07120 & $<.0001$ & 0.00026 & 0.00026 & $<.0001$ \\
\hline Beta & 6.91080 & 7.07120 & $<.0001$ & 0.00026 & 0.00026 & $<.0001$ \\
\hline
\end{tabular}

GL: Grados de Libertad; SC: Suma de Cuadrados. Elaboración propia 
certeza estadística del $95 \%$ que los parámetros obtenidos en los tres modelos y en los ocho riegos son confiables. Al analizar en la misma tabla la medida de asimetría se puede observar para el modelo Beta en el parámetro 1, el valor $\left|g_{1 \mathrm{i}}\right|>1.0$, que lo sitúa como un parámetro con comportamiento no lineal considerable y de baja certidumbre para fines de predicción. En cuanto al modelo de Gompertz en el parámetro (k), presenta tres riegos con valor $\left|g_{1 \mathrm{i}}\right|$ $>0.25$ y cinco por debajo del mismo, mientras que el de Verhulst sólo muestra uno con valor $\left|g_{1 \mathrm{i}}\right|>0.25 \mathrm{y}$ siete $<0.25$, lo que indica para este parámetro que el modelo Verhulst es el mejor calificado, al resultar que tiene propiedades razonablemente cercanas al de una regresión lineal con mínima varianza, distribución normal y con intervalos de confianza seguros para la construcción de inferencias.

En la Tabla 5 se presentan los datos del parámetro ( $t$ i), señalan que su estimado cumple en general en los tres modelos y con todos los riegos con los límites de confianza obtenidos al $95 \%$ y la medida de asimetría fue $\left|g_{1 i}\right|<0.1$ en todos los casos de riego y modelos, lo que muestra en este parámetro un comportamiento estadístico muy cercano a un modelo lineal. De este análisis de límites de confianza y asimetría de ambos parámetros se deduce que los modelos Gompertz y Verhulst son confiables y más recomendables para obtener predicciones que el modelo Beta.

En cuanto a la correlación entre parámetros, en la Tabla 6 se indica que la correlación es muy baja o no existe, ya que este coeficiente fue: $r<0.35$ en el modelo de Gompertz, en Verhulst: $r<0.012$ y en Beta: $r<0.15$, por lo que la contribución al resultado del modelo de cada uno de los parámetros es independiente.

Elaboración propia.

Elaboración propia.
Tabla 4. Parámetros $(k)$ y (t m), error de estimación (Ê 95\%) y asimetría $|\mathrm{g} l i|$ de los modelos ensayados

\begin{tabular}{|c|c|c|c|c|c|c|c|c|}
\hline Modelo & Parámetro & Estimado & $\hat{\mathbf{E}} \mathbf{9 5 \%}(\mathbf{\pm})$ & $\left|\mathbf{g}_{\mathbf{1 i}}\right|$ & Parámetro & Estimado & $\hat{\mathbf{E}} \mathbf{9 5 \%} \mathbf{( \pm )}$ & $\left|\mathbf{g}_{\mathbf{1 i}}\right|$ \\
\hline Riego 1 & & & & & Riego 5 & & & \\
\hline Gompertz & $k$ & 0.5190 & 0.0179 & 0.0741 & $k$ & 0.8810 & 0.7663 & 0.2562 \\
\hline Verhulst & $k$ & 0.7429 & 0.0776 & 0.2207 & $k$ & 1.2621 & 1.1833 & 0.1211 \\
\hline Beta & $t m$ & 16.5606 & 1.5700 & 1.1544 & $t m$ & 9.0918 & 8.5301 & 1.2835 \\
\hline Riego 2 & & & & & Riego 6 & & & \\
\hline Gompertz & $k$ & 0.6640 & 0.0345 & 0.1127 & $k$ & 1.1623 & 0.1503 & 0.3053 \\
\hline Verhulst & $k$ & 0.9432 & 0.0640 & 0.1468 & $k$ & 1.6475 & 0.0860 & 0.0998 \\
\hline Beta & $t m$ & 18.9991 & 1.5623 & 1.2432 & $t m$ & 9.7916 & 1.3657 & 1.7502 \\
\hline Riego 3 & & & & & Riego 7 & & & \\
\hline Gompertz & $k$ & 0.6111 & 0.0302 & 0.1030 & $k$ & 1.2003 & 0.1120 & 0.1474 \\
\hline Verhulst & $k$ & 0.8648 & 0.0943 & 0.2286 & $k$ & 1.6877 & 0.1679 & 0.1941 \\
\hline Beta & $t m$ & 14.1439 & 0.8399 & 1.1916 & $t m$ & 8.2644 & 1.2086 & 1.8268 \\
\hline Riego 4 & & & & & Riego 8 & & & \\
\hline Gompertz & $k$ & 0.7407 & 0.0499 & 0.1419 & $k$ & 0.8588 & 0.2806 & 0.5817 \\
\hline Verhulst & $k$ & 1.0544 & 0.0464 & 0.0930 & $k$ & 1.1655 & 0.2885 & 0.4269 \\
\hline Beta & $t m$ & 14.8940 & 0.9696 & 1.2800 & $t m$ & 7.0997 & 0.9323 & 1.6977 \\
\hline
\end{tabular}

Tabla 5. Parámetro (ti), error de estimación (Ê 95\%) y asimetría |g li | de los modelos ensayados

\begin{tabular}{|c|c|c|c|c|c|c|}
\hline Modelo & Estimado & $\hat{\mathbf{E}} \mathbf{9 5 \%}(\mathbf{\pm})$ & $\left|\mathbf{g}_{\mathbf{1 i}}\right|$ & Estimado & $\hat{\mathbf{E}} \mathbf{9 5 \%} \mathbf{( \pm )}$ & $\left|\mathbf{g}_{\mathbf{1 i}}\right|$ \\
\hline Riego 1 & & & & Riego 5 & & \\
\hline Gompertz & 5.6531 & 0.0563 & $(0.0079)$ & 5.0877 & 0.1251 & $(0.0242)$ \\
\hline Verhulst & 6.5379 & 0.1590 & $(0.0002)$ & 5.6224 & 0.0562 & $(0.0001)$ \\
\hline Beta & 4.7899 & 2.2036 & $(0.0588)$ & 6.2604 & 0.4841 & $(0.1684)$ \\
\hline Riego 2 & & & & Riego 6 & & \\
\hline Gompertz & 8.8062 & 0.0663 & $(0.0119)$ & 4.0556 & 0.0924 & $(0.0368)$ \\
\hline Verhulst & 9.4883 & 0.0817 & 0.0000 & 4.4475 & 0.0364 & 0.0003 \\
\hline Beta & 9.7838 & 1.8824 & $(0.0365)$ & 4.0923 & 1.8080 & $(0.6689)$ \\
\hline Riego 3 & & & & Riego 7 & & \\
\hline Gompertz & 7.6156 & 0.0685 & $(0.0110)$ & 3.3715 & 0.0656 & $(0.0243)$ \\
\hline Verhulst & 8.3984 & 0.1432 & $(0.0000)$ & 3.7548 & 0.0669 & $(0.0024)$ \\
\hline Beta & 9.3104 & 0.7895 & $(0.1900)$ & 3.4934 & 1.5496 & $(0.6871)$ \\
\hline Riego 4 & & & & Riego 8 & & \\
\hline Gompertz & 8.2494 & 0.0771 & $(0.0148)$ & 2.9294 & 0.8687 & $(0.0608)$ \\
\hline Verhulst & 8.8789 & 0.0475 & $(0.0000)$ & 3.4402 & 0.2376 & $(0.0021)$ \\
\hline Beta & 9.8627 & 0.9962 & $(0.2134)$ & 3.3751 & 0.8421 & $(0.6518)$ \\
\hline
\end{tabular}


Tabla 6. Matriz de correlación entre parámetros de los modelos

\begin{tabular}{|c|c|c|c|c|}
\hline \multirow{2}{*}{ Riego } & \multirow{2}{*}{ Parámetro } & Gompertz & Verhulst & Beta \\
\cline { 3 - 5 } & & Parámetro $\boldsymbol{k}$ & Parámetro $\boldsymbol{k}$ & Parámetro $\boldsymbol{t} \boldsymbol{m}$ \\
\hline 1 & $+i$ & 0.3191 & 0.0015 & $(0.2908)$ \\
\hline 2 & $+i$ & 0.3192 & 0.0000 & $(0.0177)$ \\
\hline 3 & $+i$ & 0.3191 & 0.0000 & 0.0947 \\
\hline 4 & $+i$ & 0.3187 & 0.0000 & 0.1496 \\
\hline 5 & $+i$ & 0.3197 & 0.0004 & 0.1106 \\
\hline 6 & $t i$ & 0.3501 & $(0.0031)$ & $(0.1163)$ \\
\hline 7 & $t i$ & 0.2885 & 0.0123 & $(0.1430)$ \\
\hline 8 & $t i$ & 0.3165 & 0.0087 & $(0.3498)$ \\
\hline
\end{tabular}

Elaboración propia.

Tabla 7. Indicadores de bondad de ajuste de los modelos

\begin{tabular}{|c|c|c|c|c|c|c|c|}
\hline Modelo & MEA* & $M C E^{* *}$ & $R^{2}$ & Modelo & MEA* & $M C E^{* *}$ & $R^{2}$ \\
\hline \multicolumn{4}{|c|}{ Riego 1} & \multicolumn{4}{|c|}{ Riego 5} \\
\hline Gompertz & 0.0063 & 0.0001 & 0.9995 & Gompertz & 0.0149 & 0.0040 & 0.9973 \\
\hline Verhulst & 0.0176 & 0.0007 & 0.9956 & Verhulst & 0.0074 & 0.0001 & 0.9994 \\
\hline Beta & 0.0795 & 0.0105 & 0.9350 & Beta & 0.0407 & 0.0030 & 0.9801 \\
\hline \multicolumn{4}{|c|}{ Riego 2} & \multicolumn{4}{|c|}{ Riego 6} \\
\hline Gompertz & 0.0062 & 0.0015 & 0.9993 & Gompertz & 0.0041 & 0.0000 & 0.9979 \\
\hline Verhulst & 0.0092 & 0.0002 & 0.9988 & Verhulst & 0.0016 & 0.0000 & 0.9996 \\
\hline Beta & 0.1005 & 0.0167 & 0.9172 & Beta & 0.0358 & 0.0021 & 0.9118 \\
\hline \multicolumn{4}{|c|}{ Riego 3} & \multicolumn{4}{|c|}{ Riego 7} \\
\hline Gompertz & 0.0076 & 0.0001 & 0.9993 & Gompertz & 0.0005 & 0.0000 & 0.9989 \\
\hline Verhulst & 0.0168 & 0.0006 & 0.9969 & Verhulst & 0.0005 & 0.0000 & 0.9988 \\
\hline Beta & 0.0646 & 0.0071 & 0.9648 & Beta & 0.0050 & 0.0000 & 0.9273 \\
\hline \multicolumn{4}{|c|}{ Riego 4} & \multicolumn{4}{|c|}{ Riego 8} \\
\hline Gompertz & 0.0098 & 0.0020 & 0.9990 & Gompertz & 0.0003 & 0.0000 & 0.9798 \\
\hline Verhulst & 0.0062 & 0.0001 & 0.9996 & Verhulst & 0.0002 & 0.0000 & 0.9890 \\
\hline Beta & 0.0786 & 0.0107 & 0.9478 & Beta & 0.0004 & 0.0000 & 0.9717 \\
\hline
\end{tabular}

*MEA (media del error absoluto); ${ }^{* *}$ MCE (media del cuadrado del error). Elaboración propia.

Se observa también que consistentemente este modelo presenta el menor valor de ese indicador. Para el caso de la MEA y MCE en los riegos 1, 2 y 3 , se aprecia que el modelo Gompertz presenta los valores menores, lo cual es deseable; mientras que el Verhulst en los riegos 4, 5, 6 y 8 son los más bajos. En cambio, para el riego 7 se aprecia similitud en Gompertz y Verhulst. Para todos los indicadores, el Beta es el menos recomendable al comparase con los otros dos modelos. De acuerdo a este análisis, sería indistinto recomendar Gompertz o Verhulst para utilizarse como modelo de predicción en índices de riego.

Para validar los supuestos de normalidad e independencia del modelo se realizó un examen del residual, se practicó la prueba de las rachas (run test) para comprobar la independencia de los errores; esto se muestra en la Tabla 8, en donde se describe que el modelo Gompertz presenta independencia entre errores en cuatro riegos, el de Verhulst en tres y Beta en uno.

En cuanto a la verificación de normalidad con la prueba de Shapiro-Wilk presentada en la Tabla 9, indica que el modelo de Gompertz mostró características de distribución normal del residual en cinco riegos, el de Verhulst en cuatro y el Beta en siete.

Con base en los distintos análisis expresados y resumidos en la Tabla 10, tanto el modelo Gompertz como Verhulst muestran condiciones estadísticas aceptables para ser utilizados como predictores de índices de riego, cuyos parámetros se muestran en las Tablas 4 y 5, siendo las ecuaciones:

Modelo logístico de Verhulst: $y=\frac{y \max }{1+e^{-k(t-t i)}}(1)$

Función Gompertz: $\quad y=y \max e^{-e^{-k(t-t i)}}$ 
Tabla 8. Prueba de las rachas para independencia de los errores $\alpha=0.05$

\begin{tabular}{|c|c|c|c|c|c|c|c|c|c|}
\hline \multirow{2}{*}{ Riego } & \multicolumn{3}{|c|}{ Gompertz } & \multicolumn{3}{|c|}{ Verhulst } & \multicolumn{3}{|c|}{ Beta } \\
\hline & $r$ & $r$ crítico & se acepta & $r$ & r crítico & se acepta & $r$ & r crítico & se acepta \\
\hline 1 & 5 & 4 & $\mathrm{HO}$ & 3 & 3 & $\mathrm{Hl}$ & 4 & 6 & $\mathrm{Hl}$ \\
\hline 2 & 5 & 5 & $\mathrm{HI}$ & 5 & 3 & $\mathrm{HO}$ & 4 & 7 & $\mathrm{Hl}$ \\
\hline 3 & 5 & 3 & $\mathrm{HO}$ & 3 & 2 & $\mathrm{HO}$ & 4 & 4 & $\mathrm{Hl}$ \\
\hline 4 & 3 & 2 & $\mathrm{HO}$ & 5 & 5 & $\mathrm{HI}$ & 2 & 5 & $\mathrm{HI}$ \\
\hline 5 & 3 & - & - & 4 & 2 & $\mathrm{HO}$ & 4 & 2 & $\mathrm{HO}$ \\
\hline 6 & 3 & - & - & 0 & - & - & 2 & 3 & $\mathrm{Hl}$ \\
\hline 7 & 5 & 2 & $\mathrm{HO}$ & 0 & - & - & 2 & 2 & $\mathrm{HI}$ \\
\hline 8 & 5 & - & - & 4 & - & - & 5 & - & $\mathrm{HI}$ \\
\hline
\end{tabular}

Elaboración propia.

Tabla 9. Prueba de normalidad Shapiro-Wilk (W) $\alpha=0.05$

\begin{tabular}{|c|c|c|c|}
\hline \multicolumn{2}{|c|}{ Riego $1(n=21, W t=0.908)$} & \multicolumn{2}{|c|}{ Riego $5(n=10, W t=0.842)$} \\
\hline Gompertz & 0.893 & Gompertz & 0.944 \\
\hline Verhulst & 0.887 & Verhulst & 0.939 \\
\hline Beta & 0.902 & Beta & 0.958 \\
\hline \multicolumn{2}{|c|}{ Riego $2(n=23, W t=0.914)$} & \multicolumn{2}{|c|}{ Riego $6(n=12, W t=0.859)$} \\
\hline Gompertz & 0.757 & Gompertz & 0.858 \\
\hline Verhulst & 0.852 & Verhulst & 0.852 \\
\hline Beta & 0.956 & Beta & 0.950 \\
\hline \multicolumn{2}{|c|}{ Riego $3(n=16, W t=0.887)$} & \multicolumn{2}{|c|}{ Riego $7(n=10, W t=0.842)$} \\
\hline Gompertz & 0.905 & Gompertz & 0.851 \\
\hline Verhulst & 0.840 & Verhulst & 0.885 \\
\hline Beta & 0.950 & Beta & 0.912 \\
\hline \multicolumn{2}{|c|}{ Riego $4(n=17, W t=0.892)$} & \multicolumn{2}{|c|}{ Riego $8(n=8, W t=0.818)$} \\
\hline Gompertz & 0.941 & Gompertz & 0.881 \\
\hline Verhulst & 0.960 & Verhulst & 0.854 \\
\hline Beta & 0.958 & Beta & 0.963 \\
\hline
\end{tabular}

Elaboración propia.

Tabla 10. Resumen de indicadores estadísticos

\begin{tabular}{|c|c|c|c|}
\hline Indicadores & Gompertz & Verhulst & Beta \\
\hline ANDEVA del modelo & $P<.0001$ & $P<.0001$ & $P<.0001$ \\
\hline Parámetro 1 & $k$ & $k$ & $t m$ \\
\hline L.C. $95 \%$ & Confiable & Confiable & Confiable \\
\hline Asimetría & $\left|g_{1 \mathrm{i}}\right|<0.25$ en 5 riegos & $\left|g_{1 \mathrm{i}}\right|<0.25$ en 7 riegos & $\left|g_{1 \mathrm{i}}\right|>1.0$ en 8 riegos \\
\hline Parámetro 2 & $+i$ & $t i$ & $t i$ \\
\hline L.C. $95 \%$ & Confiable & Confiable & Confiable \\
\hline Asimetría & $\left|g_{1 \mathrm{i}}\right|<0.1$ & $\left|g_{1 i}\right|<0.1$ & $\left|g_{1 i}\right|<0.1$ \\
\hline \multicolumn{4}{|l|}{ Correlación } \\
\hline entre parámetros & $<0.35$ & $<0.012$ & $<0.15$ \\
\hline \multicolumn{4}{|l|}{ Bondad de Ajuste } \\
\hline MEA & $<.01153$ & $<.01536$ & $<.07194$ \\
\hline MCE & $<0.00015$ & $<.00057$ & $<.0048$ \\
\hline$R^{2}$ & 0.98 a 0.99 & 0.98 a 0.99 & 0.91 a .98 \\
\hline \multicolumn{4}{|l|}{ Examen del Residual } \\
\hline Rachas & Independencia en 4 riegos & Independencia en 3 riegos & Independencia en 1 riego \\
\hline Shapiro - Wilk & Normalidad en 5 riegos & Normalidad en 4 riegos & Normalidad en 7 riegos \\
\hline
\end{tabular}



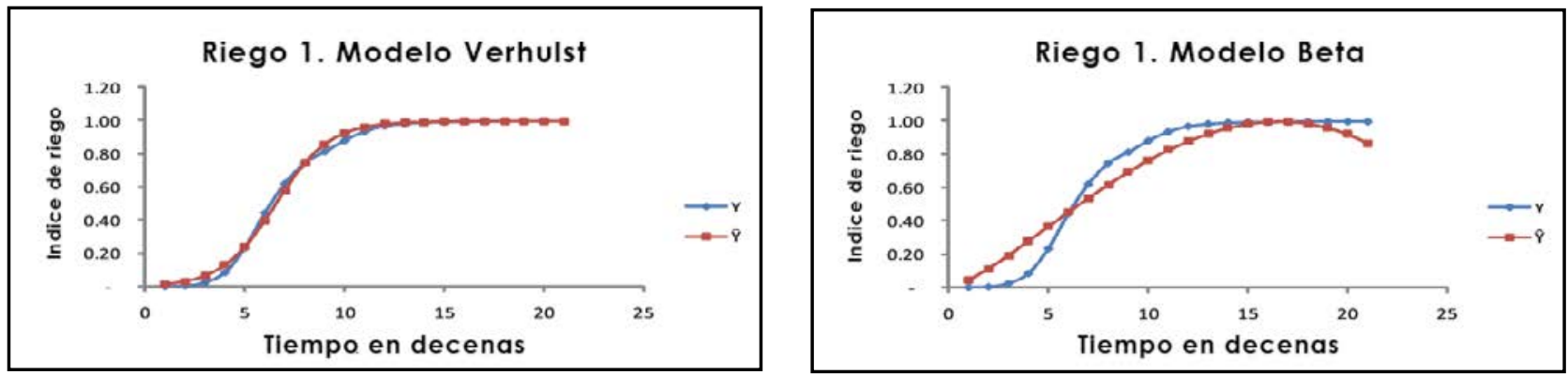

Figura 2. Indicadores de riego estimados y observados del Riego 1 en los modelos Verhulst y Beta. Elaboración propia.
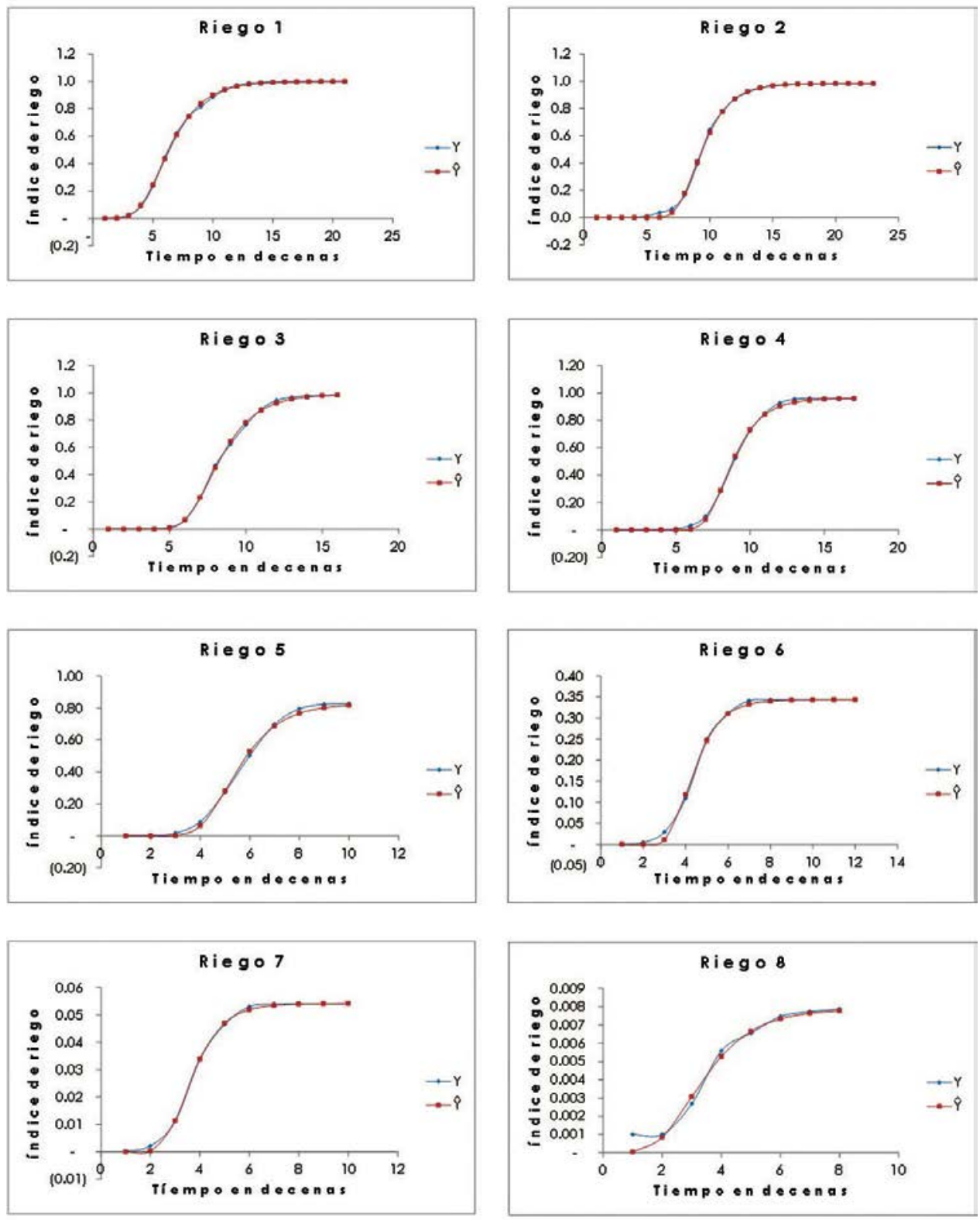

Figura 3. Índices de riego observados y estimados con el modelo Gompertz. Elaboración propia. 
En la Figura 2 se presenta el comportamiento típico que reflejaron los modelos Verhulst y Beta; en donde puede apreciarse que en el segundo la expresión gráfica no es una curva sigmoide, aunque algunos indicadores de ajuste fueron buenos. Se muestra además el comportamiento del modelo Gompertz para los ocho riegos, y se observa en la Figura 3 una sobreposición muy estrecha entre las curvas de datos observados y los normales estimados.

\section{DISCUSIÓN}

La información analizada sobre la superficie regada y acumulada del estudio fue recabada y registrada por los operadores del sistema de entrega de agua a los usuarios de los módulos de riego, por lo que los datos provienen de registros históricos no controlados. Por ello, en la investigación se dio énfasis al análisis estadístico y a la aplicación de las pruebas no paramétricas por tratarse de modelos no lineales y para evitar conclusiones erróneas, aun cuando siempre se corre el riesgo. Al respecto, en la Tabla 1 se presentan aplicaciones del primer riego después de enero en aproximadamente 1,800 ha, que representan $2.9 \%$ de la superficie total establecida, lo cual puede indicar que se reporta información tardía, lo que afecta la toma de decisiones en el manejo del agua dentro de la presa Morelos que recibe agua del caudal del río Colorado (Figura 4).

La representación gráfica del riego en la Figura 1 permite interpretar el avance del riego en forma objetiva, identificar intervalos entre los riegos y detectar atrasos en los programas para realizar los ajustes requeridos. La expresión de la superficie regada acumulada en fracción de la superficie total establecida (índices de riego), como se presenta en la Tabla 2, facilita extrapolar los datos para la programación de riego al obtenerse los índices con los modelos logrados en el estudio (Ecuaciones 1 y 2).

Al conocer el comportamiento gráfico de la superficie regada acumulada en curvas sigmoides que pueden ser simuladas con funciones de crecimiento, fue posible comparar y seleccionar el mejor ajuste de los modelos ensayados (Tablas 4 y 5), lo cual fue el principal objetivo de esta investigación y da pauta para modelar en otros cultivos con la metodología propuesta.

El empleo de regresiones no lineales cuando se conocen las funciones matemáticas que los simulan facilita la obtención de los parámetros de los modelos

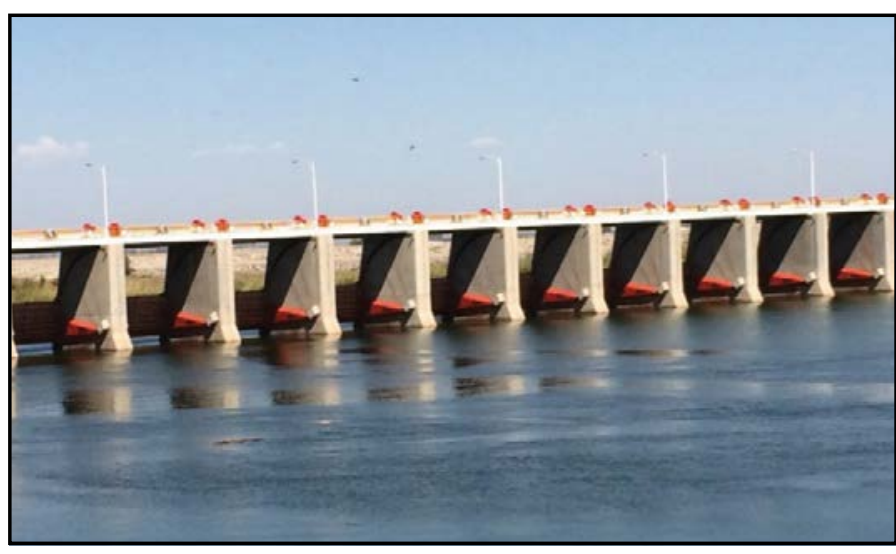

Figura 4. Presa Morelos, obra hidráulica de recepción de agua del río Colorado. Fotografía proporcionada por Carlos H. Castro González.

siempre y cuando se fundamenten estadísticamente, más aún cuando la variable independiente es el tiempo como en este estudio y puede presentar correlación entre residuos y que aparentemente se tenga un buen modelo pero que no permita obtener predicciones acertadas.

Para el caso de los modelos empleados, el análisis estadístico aplicado permitió obtener modelos confiables para las condiciones ensayadas. La modelación de la superficie regada acumulada expresada en índices de riego se realizó en forma independiente de cada uno de los riegos; es decir, el tiempo de inicio en todos los riegos fue la Decena 1, como se muestra en la Tabla 2 y Figuras 2 y 3 . Se procedió así en virtud de que el objetivo principal del estudio fue seleccionar el modelo de mejor ajuste y con la pretensión de utilizarlo en modelaciones futuras con otros cultivos. Será oportuno utilizar esta metodología con la información de varios ciclos agrícolas y en distintos cultivos para obtener los índices de riego de la cédula completa del Distrito de Riego 014.

CONCLUSIONES

Los modelos Gompertz y Verhulst permitieron estimar estadísticamente los índices de riego con fines de predicción, por lo que esta metodología puede ser utilizada para la elaboración de planes de riego en el cultivo de trigo en la región de los valles de Mexicali y San Luis Río Colorado. Esto se afirma considerando que fueron los modelos que cumplieron con las restricciones estadísticas establecidas en este estudio. Se recomienda el análisis y obtención de índices de riego empleando información de un mayor número de ciclos de cultivo. 
- ALONSO BÁEZ, M. et al. Modelo logístico: herramienta para diagnosticar el cuánto y cuándo fertirrigar. Terra Latinoamericana, 21 (2): 225-231, 2003.

- $\quad$ BIRCH, C. A new generalized logistic sigmoid growth equation compared with the Richards growth equation. Annals of Botany, 83(6): 713-723, 1999.

- CONAGUa (COMISIÓN NACIONAL DEL AGUA). Capítulo II. De la operación. Anexos del título de concesión (ATC). Distrito de Riego Río Colorado SRL de IP. México: Autor, 2009.

- conagua (COMISIÓN NACIONAL DEL AGUA). Programa temporal de renta de permisos de riego para enfrentar los efectos del terremoto del 4 de abril de 2010 en el Valle de Mexicali. Documento interno. Mexicali, B. C., México: Autor, 2010.

- CORTez larA, A. A. Gestión local y binacional del agua del río Colorado: El reto de la región fronteriza, CaliforniaBaja California. En A. Cortez Lara, S. Whiteford \& M. Chávez (Coords.), Seguridad, agua y desarrollo: el futuro de la Frontera México-Estados Unidos, Tijuana, B. C. (333-364). El Colegio de la Frontera Norte-Michigan State University, 2005.
- CORTEZ larA, A. A. Gestión y manejo del agua: el papel de los usuarios agrícolas del Valle de Mexicali. Revista Problemas del Desarrollo, 42(167): 71-95, 2011.

- KIRCHHOFF, W. H. Logistic function profile fit: A least-squares program for fitting interface profiles to an extended logistic function. Journal of Vacuum Science \& Technology, 30(5): 33 55,2012

- $\quad$ Plan de riegos, sistema de gravedad (PRSG) 2010-2011. Distrito de Riego Río Colorado SRL de IP de CV. Mexicali, B. C., México, 2011.

- SÁNCHEZ, M. V. Introducción. En V. Sánchez (Coord.), El revestimiento del canal todo americano. ¿ Competencia o cooperación por el agua en la frontera México-Estados Unidos? (13-23). Tijuana, B. C., México: El Colegio de la Frontera Norte-Plaza y Valdés, 2004.

- SRE (SECRETARÍA DE RELACIONES EXTERIORES). Tratado sobre distribución de aguas internacionales entre Estados Unidos Mexicanos y Estados Unidos de Norteamérica (85-114). México: Autor, 1944.

- YIN, X. et al. A flexible sigmoid function of determinate growth. Annals of Botany, 91 (3): 361-371, 2003. 